\title{
Architecture of Signature miRNA Regulatory Networks in Cancer Chemoprevention
}

\author{
Sujit Nair • Ah-Ng Tony Kong
}

Published online: 11 January 2015

(C) Springer International Publishing AG 2015

\begin{abstract}
With new high-throughput technologies and superior computational power available for application to current pharmacology research, biomarker discovery has probably entered its most exciting phase to date, especially with the concurrent advent of systems network biology for "big data." Study of recurrent network motifs in network architecture can inform us better about regulatory pathways in the cellular milieu, more so in complex disease states like cancer. In this review, we focus on the architecture of miRNA networks with emphasis on chemoresistance networks in response to chemotherapeutic drugs, chemoprevention networks modulated by dietary phytochemicals, and novel bifunctional networks comprised of bifunctional miRNAs that operate in both chemoresistance and chemoprevention. Since miRNA cancer networks are very complex, the regulatory architecture in chemoresistance and/or chemoprevention may likely include added dimensions of modulation by epigenetic miRNAs and lncRNAs, which may explain, at least in part, the bifunctionality associated with signature miRNA nodes in these networks in addition to temporal dynamics, spatial localization, and stress conditions in the dynamic networks representing the complex cellular milieu. Collectively, by a perusal of our chemoresistance,
\end{abstract}

This article is part of the Topical Collection on miRNA and Cancer Prevention and Therapeutic Agents

S. Nair $(\bowtie)$

Amrita Cancer Discovery Biology Laboratory and Amrita School of

Pharmacy, Amrita Vishwa Vidyapeetham University, AIMS

Ponekkara P.O, Kochi, Kerala 682 041, India

e-mail: sujit108@gmail.com

A.-N. T. Kong

Center for Cancer Chemoprevention Research and Department of

Pharmaceutics, Ernest Mario School of Pharmacy, 160

Frelinghuysen Road, Piscataway, NJ 08854, USA chemoprevention, and bifunctional networks, we can gain deeper insights into the architecture of signature miRNA regulatory networks in cancer that will serve as the basis for future dynamic network studies and facilitate the discovery of novel miRNA/target biomarkers for preventive and/or therapeutic intervention in cancer.

Keywords Big data - Biomarker - Bifunctional network . Cancer - Chemoprevention - Chemoresistance - miRNA miRNA signature $\cdot$ Network $\cdot$ Network motif . Systems biology

$\begin{array}{ll}\text { Abbreviations } \\ \text { AITC } & \text { Allyl isothiocyanate } \\ \text { ATRA } & \text { All trans retinoic acid } \\ \text { CoMi } & \text { Context-specific miRNA regulation } \\ \text { DATS } & \text { Diallyl trisulfide } \\ \text { DIM } & \text { 3,3'-Diindolyl methane } \\ \text { EGCG } & \text { Epigallocatechin-3-gallate } \\ \text { FBL } & \text { Feedback loops } \\ \text { FFL } & \text { Feed-forward loops } \\ \text { HRPC } & \text { Hormone refractory prostate cancers } \\ \text { I3C } & \text { Indole-3-carbinol } \\ \text { Jak/STAT } & \text { Janus kinase/Signal transducer and activator of } \\ & \text { transcription } \\ \text { lncRNA } & \text { Long noncoding RNA } \\ \text { MDRL } & \text { Mitochondrial dynamic related lncRNA } \\ \text { mirDREM } & \text { MIRna Dynamic Regulatory Events Miner } \\ \text { miRNA } & \text { MicroRNA } \\ \text { NFATC3 } & \text { Nuclear factor of activated T cell isoform c3 } \\ \text { PEITC } & \text { Phenethyl isothiocyanate } \\ \text { SCC } & \text { Squamous cell carcinoma } \\ \text { TP } & \text { Tumor protein } \\ \text { TRPC5 } & \text { Transient receptor potential channel C5 }\end{array}$




\section{Introduction}

Despite substantial progress in understanding the cancer signaling network, effective therapies remain scarce due to insufficient disruption of oncogenic pathways, chemoresistance, and drug-induced toxicity $[1 \bullet \cdot$. Defects in the DNA damage response and deregulation of microRNAs (miRNAs) are important hallmarks of human cancer; hence, a full understanding of the mechanisms underlying the connection between miRNAs and DNA damage response and DNA repair pathways will positively impact our knowledge on human tumor biology and on different responses to distinct drugs [2]. By definition, miRNAs are small noncoding endogenous regulatory RNAs that fine tune gene expression in a wide range of biological processes and diseases, and exert their function by targeting $\mathrm{mRNAs}$ to trigger their degradation or inhibit protein translation [3•]. According to current estimates, most human genes are harboring miRNAs and/or are regulated by them; thus, miRNAs can form complex regulatory networks by themselves, but because their expression is often tightly coordinated with gene expression, they form an intertwined regulatory network with many possible interactions among gene and miRNA regulatory pathways [4]. Indeed, identifying master regulators of biological processes and mapping their downstream gene networks are key challenges in systems biology that remain to be overcome [5]. Although initially seen as a very promising source of breakthroughs in cancer management, there has been little translation of miRNA science from the bench to the bedside, thus underscoring the need to highlight the potential role of miRNAs in cancer prevention, viz., their use as biomarkers and as targets for chemoprevention [6]. Given their significance in modulating gene expression, miRNA research can provide insight into the pleiotropic biological effects that chemopreventive agents often display and a deeper understanding of their mechanism(s) of action to inhibit carcinogenesis [7]. In this review, we focus on the architecture of miRNA networks with emphasis on three kinds of important networks in cancer, viz., chemoresistance networks in response to chemotherapeutic drugs, chemoprevention networks modulated by dietary phytochemicals, and novel bifunctional networks comprised of bifunctional miRNAs that operate in both chemoresistance and chemoprevention.

\section{Systems Biology of microRNA Cancer Networks}

The biological sciences are in a state of rapid development, driven largely by the new technologies that have developed as byproducts of the Human Genome Project; thus, the true revolution inspired by genomics is probably changing what was exclusively a laboratory science into an information science [8]. Rather than focusing exclusively on single drug targets, systems pharmacology examines the holistic response of a phenotype-dependent pathway or pathways to drug perturbation [9]. "Top-down" systems biology identifies molecular interaction networks on the basis of correlated molecular behavior observed in genome-wide "omics" studies, whereas "bottom-up" systems biology examines the mechanisms through which functional properties arise in the interactions of known components [10]. Importantly, data integration efforts can be achieved through the conversion of data from the various datasets of recent large-scale projects into singlenode-type networks, gene-set libraries, or multipartite graphs resulting in a lean "big data" integration strategy that could bring us closer toward the goal of realizing personalized medicine [11].

miRNA Network Biology and Cancer Biomarker Discovery

We have recently reported [12••] on our use of systems pharmacology for elucidation of differential signaling regulatory networks governing hormone refractory prostate cancers (HRPC) and identification of putative "target hubs" in the architecture of these gene networks. In addition, we have constructed [13] transcription factor networks to elucidate potential relationships between NRF2 (NFE2L2) and NFkB1 in the etiopathogenesis of inflammation and cancer. Similarly, it is known that the miRNA network is linked at several and unexpected levels with cancer-related signaling pathways; thus, our understanding of the role(s) and regulation of the miRNA network has been extended to include classical cell signaling, i.e., the miRNA network complements cell signaling in cancer [14]. Recently, high-throughput sequencing coupled with network analysis [15] was used to identify cancer-specific miRNA signatures and potentially useful biomarkers in colorectal cancer. Zhang et al. [16] reconstructed the human miRNA-mRNA interaction network exhibiting scale-free features and designed a novel cancer miRNA biomarker prediction framework applied to prostate cancer study that could also be extended to other cancers. Further, Zhu et al. [17] used network biology for predictive marker discovery using context-specific miRNA regulation (CoMi) patterns to represent a distinctive feature of the miRNA regulatory network in the transcriptome. Indeed, the utility of miRNAs as prognostic biomarkers and possible druggable target(s) for circumventing multidrug resistance in cancer chemotherapy [18] is now being greatly appreciated.

Architecture of miRNA Networks in Cancer

Emerging models supporting an miRNA-modulated systems-level or network-wide regulation of gene expression are exciting and will yield deep insight into the regulatory architecture of biology; however, because of the technical challenges facing the network-based study of miRNAs, 
many gaps remain in our understanding [19]. Network motifs are subgraphs that are statistically overrepresented within networks [20]; hence, recurring network motifs may have important gene regulatory role(s). Interestingly, transcription factors and miRNAs can jointly regulate target gene expression in the forms of feed-forward loops (FFLs) or feedback loops (FBLs), which serve as important motifs in gene regulatory networks [21] and play critical roles in multiple biological processes and different diseases. The proteins encoded by the genes targeted by miRNAs may act as key components of cellular networks; thus, the use of biological molecular network information for the purposes of elucidating the role of miRNAs in molecular disease mechanisms is a key objective in systems biomedicine [3•]. Further, because miRNAs can modulate epigenetic architecture and can be regulated by epigenetic alteration, they can reasonably play an important role(s) in mediating the crosstalk between epigenetic regulators; thus, study of the epigenetic-miRNA regulatory pathway is a promising avenue for the design of innovative strategies in the fight against human cancer [22].

\section{microRNAs and Chemoresistance Networks}

One of the most important factors limiting the effectiveness of chemotherapy is the primary and secondary resistance of cancer cells; thus, understanding the genetic factors and mechanisms that contribute to the lack of or low sensitivity of tumor tissue to cytostatics is a key element in the currently developing trend of personalized medicine [23]. There is known to be great heterogeneity in tumors with chemoresistance [24], which makes the disease far more difficult to treat using conventional chemotherapy acting on limited known targets. Indeed, drug resistance is a complex multistep process resulting from deregulated expression of many molecules, including tumor suppressor genes, oncogenes, and miRNAs [25]. A growing body of evidence suggests that miRNA polymorphisms are associated with drug metabolism and chemoresistance and that differentially expressed miRNAs play critical roles in the prediction of sensitivity to chemotherapeutic agents [26]. Experimental evidence demonstrates that dysregulation of specific miRNAs leads to drug resistance in different cancers, and correction of these miRNAs using miRNA mimics or antagomirs can normalize the gene regulatory network and signaling pathways and sensitize cancer cells to chemotherapy $[1 \bullet \bullet]$. Thus, current evidence strongly reinforces the case for a better understanding of drug resistance-related miRNAs that may eventually lead to optimized therapeutic strategies for cancer patients [25].
Fig. 1 Architecture of chemoresistance, chemoprevention, and bifunctional cancer networks showing: a putative canonical interactions between miRNAs involved in chemoresistance, b epistemologic interactions between chemoresistance miRNAs and targets, $\mathbf{c}$ putative canonical interactions between miRNAs involved in chemoprevention, $\mathbf{d}$ epistemologic interactions between chemoprevention miRNAs and targets, e putative canonical interactions between miRNAs involved in cancer bifunctionality, and $\mathbf{f}$ epistemologic interactions between bifunctional cancer miRNAs and targets. Putative canonical miRNA interactions were constructed using Cytoscape 3.1.1 [125, 126], whereas epistemologic miRNA-target interactions were delineated by Ingenuity Pathway Analysis (IPA, http://www.ingenuity.com). The bifunctional cancer network E comprised a subset of miRNAs that were common to both chemoresistance and chemoprevention networks a and c, respectively

miRNAs Involved in Chemoresistance

Against Chemotherapeutic Drugs

In our previous report [27••] on emerging role(s) for miRNA cancer signatures, we had succinctly tabulated various chemotherapeutics along with up- or downregulated miRNAs involved in resistance against them. Building on this, we reviewed 24 chemotherapeutic agents including 5fluorouracil [28-33], bortezomib [34], camptothecin [35], cetuximab [36, 37], cisplatin [38, 39], dasatinib [40], daunorubicin [41], docetaxel [39, 42, 43], doxorubicin [44-47], erlotinib [48], everolimus [49], fludarabine [50, 51], fulvestrant [52], gefitinib [53-55], gemcitabine [56-58], imatinib [59], lapatinib [55], methotrexate [60, 61], paclitaxel [38, 62], sorafenib [63], sunitinib [64], tamoxifen [65], temozolomide [66-68], and trastuzumab [69], and miRNAs involved in drug resistance to these agents. These miRNAs are included in Fig. 1a that depicts the architecture of our proposed canonical chemoresistance network showing putative interactions between 55 miRNA nodes or vertices involved in chemoresistance and 2970 undirected edges facilitating these interactions. Further, we queried these miRNAs for interactions with targets to construct an epistemologic chemoresistance network showing "arcs," i.e., directed edges, connecting miRNA nodes of the network (Fig. 1b).

\section{Architecture of Chemoresistant miRNA Networks}

miRNAs are involved in many regulatory pathways some of which are complex networks enriched in regulatory motifs like positive or negative FBLs or coherent and incoherent FFLs [70]. A methylation-based regulatory network for miR-320a in chemoresistant breast cancer through targeting of transient receptor potential channel C5 (TRPC5) and nuclear factor of activated T cells isoform c3 (NFATC3) has recently been reported [71]. The existence of FBLs between E2F1 and miRNAs have been reported [72] resulting in a complex regulatory network. Vera et al. [73] performed 


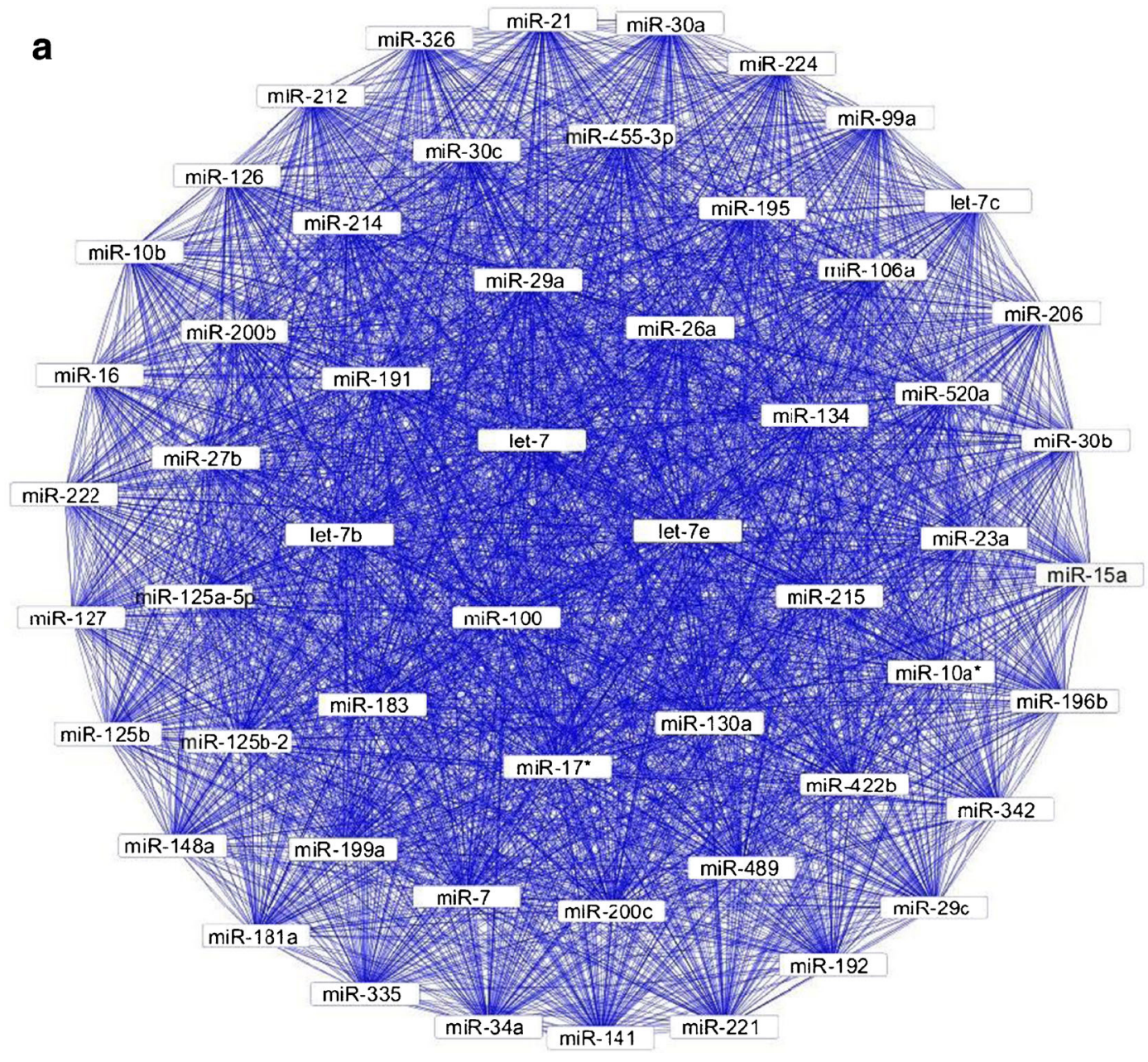

b CEP85L

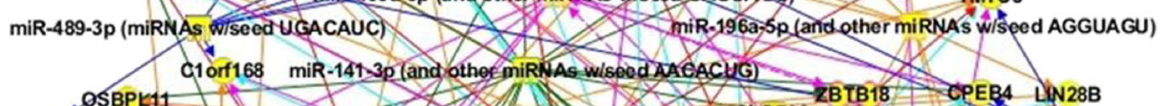

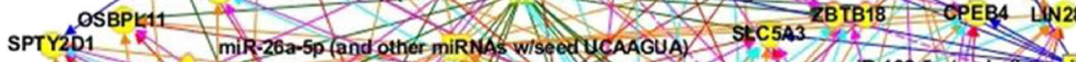

miR-192-5p (and othermiRNAs w/seed UGACCUA)

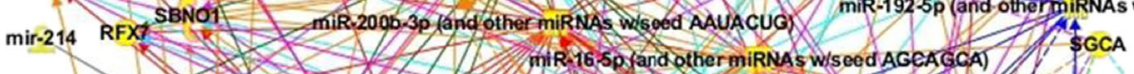

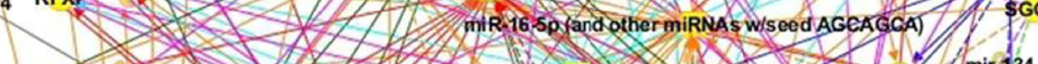

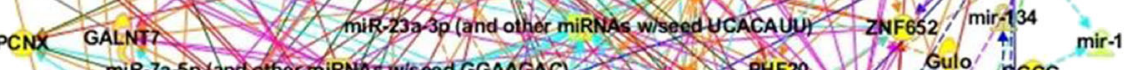

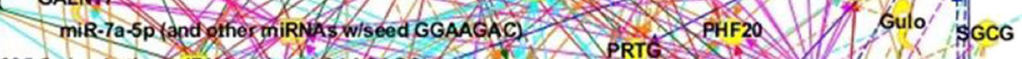

miR-335-5p (and other miRNAs wiseed CAAGAGC) INRC6B miR-30c-5p landothor thiRNÁs w/seed GWAAACA)

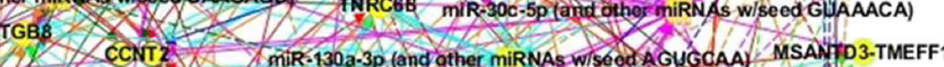

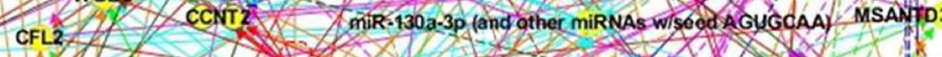

FAM167B CFL2 1 MiR-29b-3p (and other miR As wiseed AGCACCA) - TMEM1708 mir-8 mir-192

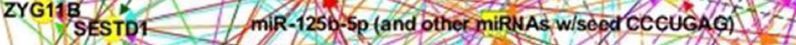
W 1 miR-34.5

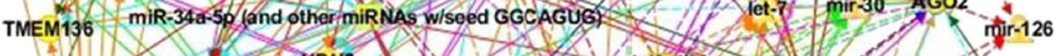

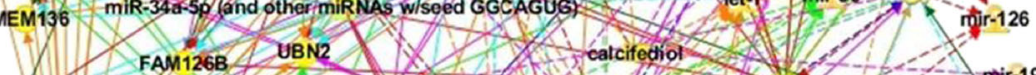

1. FAM126B miR-455-3p (miRNAs Wiseed CAGUCCA) miR 342-3p (miRNAs W/seoctCUCÁCAC) mir-191

CDKLR TMEF 1 A

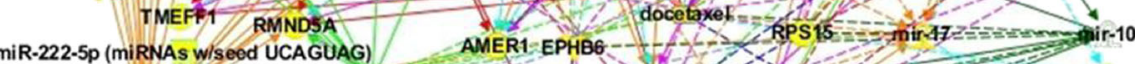
miR-21-5p (and other miRNAS w/soed A GCUUAU) TMEMB7B

miR-148b-3p (and other miRNAs w/seed CAGUGCA) - oxaliplatin mir-221 miR-10b-5p (miRNAs W/seed CCUGUAG) mir-2s

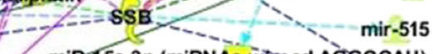
miR-15a-3p (miRNAs wi/seed AĞGCCAU) miR-100-5p (and other miRNAs w/seed ACCCGUA) mir-224 

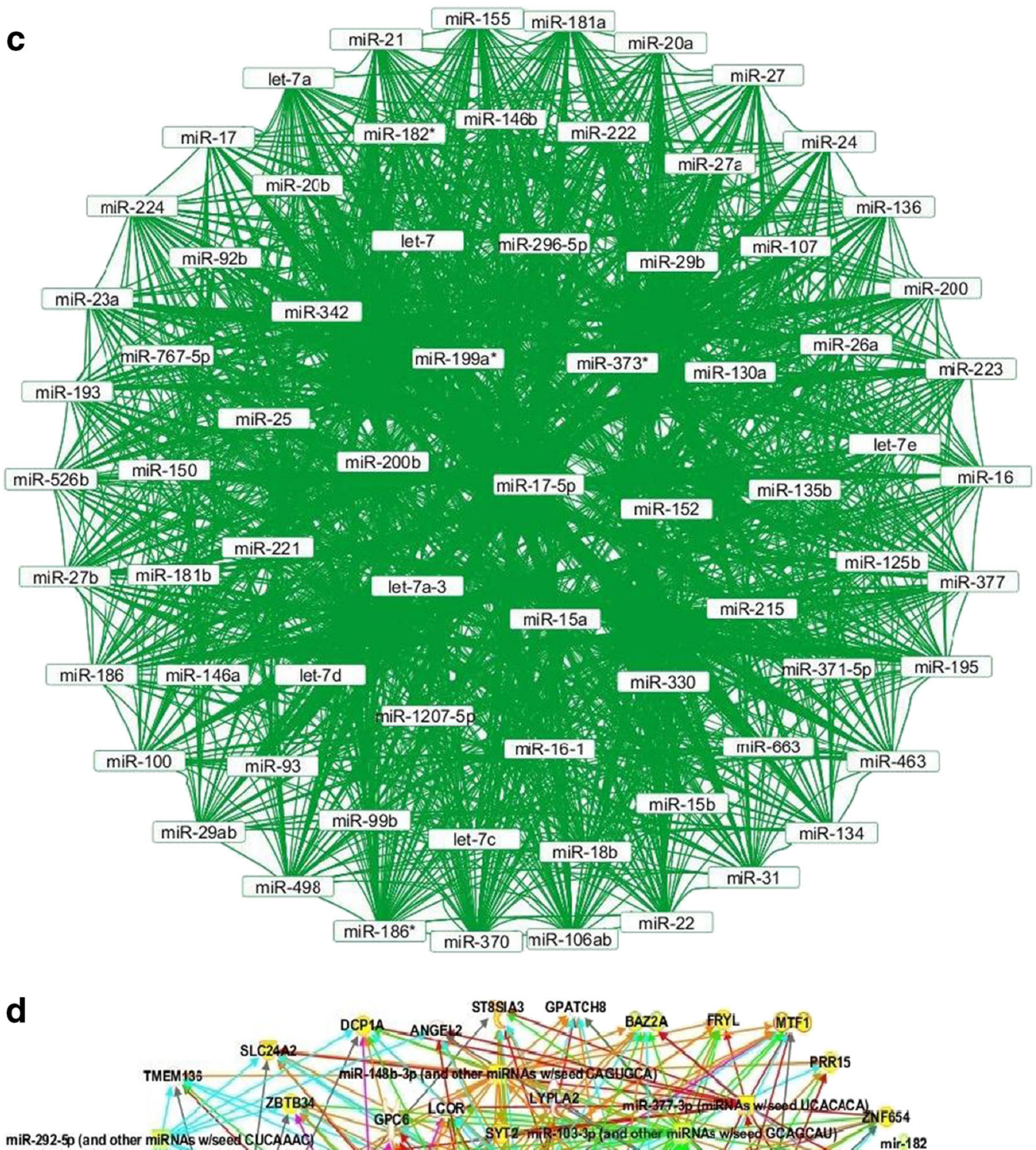

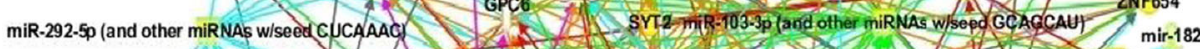

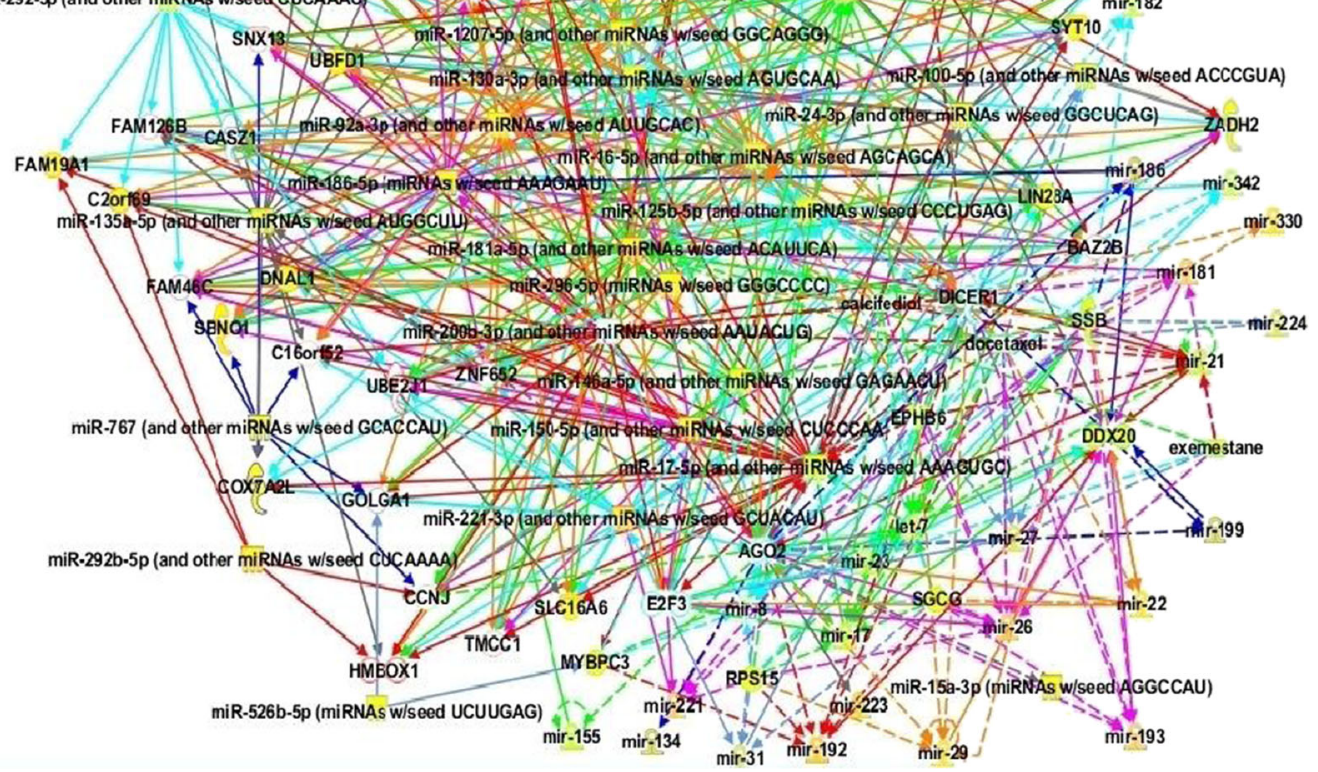

Fig. 1 (continued) 

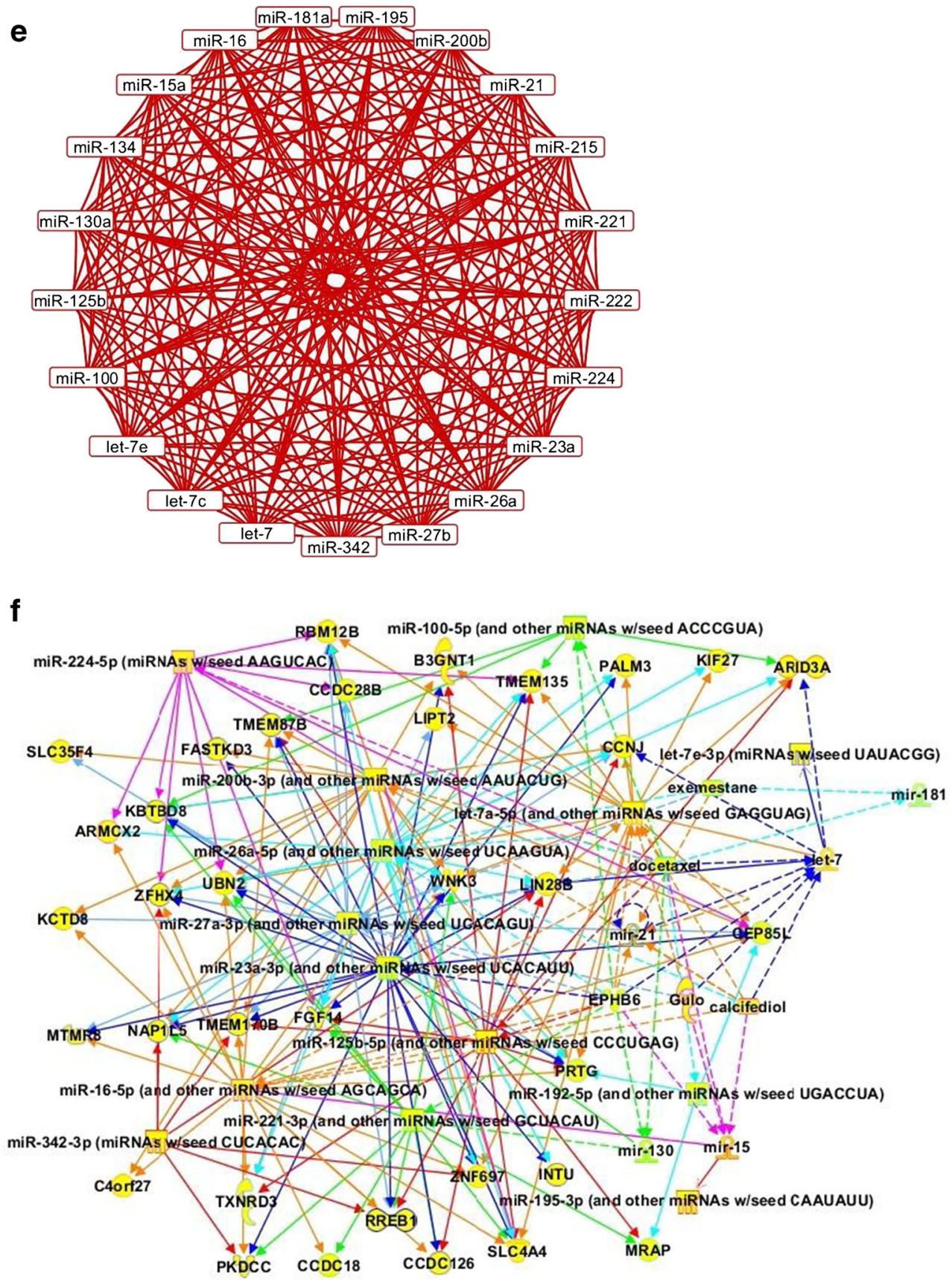

Fig. 1 (continued)

kinetic-modeling-based simulations to demonstrate that conventional genotoxic drug treatment favors selection of chemoresistant cells in genetically heterogeneous tumors, in a manner requiring dysregulation of incoherent FFLs that involve E2F1, p73/DNp73, and miR-205. Kopp et al. [74] showed that miR-200c sensitizes breast cancer cells to doxorubicin treatment by decreasing expression of receptor tyrosine kinase TrkB and the transcriptional repressor Bmil, whereas loss of miR-200c resulted in acquired chemoresistance to doxorubicin. Ratovitski [75] showed recently that cisplatin exposure of squamous cell carcinoma
SCC-11 cells led to upregulation of miR-297, miR-92b-3p, and miR-485-5p through a phosphorylated $\Delta \mathrm{Np} 63 \alpha-$ dependent mechanism and that the tumor protein (TP) p63/ miRNA functional network may play a key role in supporting the response of SCC to chemotherapy. Lecca [76] has described Bayesian methods of biological network inference for reverse engineering cancer chemoresistance mechanisms. Indeed, network-based therapy that affects network flexibility, including rewiring of network structures and focusing on "target hub" molecules in these networks, could minimize the occurrence of side effects and be a promising strategy for 
enhancing the therapeutic efficacy of cancer treatments in chemoresistance and radioresistance [77]. Based on our chemoresistance network in Fig. 1b, several miRNA nodes with high "degree," i.e., "number of interactions" were visible including miR-148b-3p, miR-21-5p, miR-10, miR-455-3p, miR-342-3p, miR-34a-5p, miR-125b-5p, miR-30, let-7, miR-29b-3p, miR-8, miR-130a-3p, miR-30c-5p, miR-3355p, miR-7a-5p, miR-23a-3p, miR-16-5p, miR-200b-3p, miR-192-5p, miR-26a-5p, miR-141-3p, miR-489-3p, miR199a-3p, among others. Further, autoregulation was observed for miR-29 and miR-29b-3p; also, let-7 and miR-30 exhibited highest "in-degree," i.e., arrows pointing towards these nodes in the directional miRNA network, whereas most other miRNA nodes exhibited higher "out-degree," i.e., arrows pointing away from these nodes in Fig. 1b. In addition, several targets of the miRNAs with high "degree" were observed in the network in Fig. $1 \mathrm{~b}$ including ZBTB18, CCNT2, AGO2, TRERF1, ETNK1, PCDH19, CCDC6, TXLNG, CEP85L, SLC5A3, TNRC6B, EPHB6, LIN28B, among others, that may be important in mediating chemoresistance via miRNAs.

\section{microRNAs and Anti-cancer Chemoprevention Networks}

Chemoprevention is a pharmacological approach to intervention in order to arrest or reverse the process of carcinogenesis [78]. We have noted earlier [79•] that mammalian cells, including human cells, respond to dietary phytochemicals by "nonclassical receptor sensing" mechanisms of electrophilic chemical-stress typified by "thiol-modulated" cellular signaling events primarily leading to the gene expression of pharmacologically beneficial effects, but sometimes unwanted cytotoxicity also. Interestingly, specific targeting of miRNAs by natural agents could open newer avenues for complete eradication of tumors by killing drug-resistant cells to improve survival outcomes in patients diagnosed with malignancies [80••]. Indeed, as previously observed [81] by us, although the precise mechanism underlying the control of miRNA expression in chemoprevention is not well understood currently, epigenetic changes could play a major role.

miRNAs Modulated by Anti-cancer Chemopreventive Agents

A summary of miRNAs modulated by several anti-cancer chemopreventive agents has been furnished by us earlier [27••]. Building on this, we reviewed the literature for 17 chemopreventive agents (and miRNAs) including all trans retinoic acid (ATRA) [82, 83], allyl isothiocyanate (AITC) [84], boswellic acid [85], calcitriol [86-88], curcumin [89-94], diallyl trisulfide (DATS) [95], 3,3'-diindolylmethane (DIM) [96, 97], ellagitannin [98], (-) epigallocatechin-3- gallate (EGCG) [99-101], genistein [96, 97, 102], green tea polyphenon-60 [103], indole-3-carbinol (I3C) [104], lycopene [105••, 106], phenethyl isothiocyanate (PEITC) [107], quercetin [108], resveratrol [109, 110], and sulforaphane [111]. These miRNAs modulated by chemopreventive agents are included in Fig. 1c that depicts the architecture of our proposed canonical chemoprevention network showing putative interactions between 71 miRNA nodes or vertices involved in eliciting the protective chemopreventive effects of these natural or synthetic dietary factors and 4969 undirected edges facilitating these interactions. Further, we queried these miRNAs for interactions with targets to construct an epistemologic chemoprevention network showing "arcs" connecting miRNA nodes of the network (Fig. 1d).

Architecture of Chemopreventive miRNA Networks

Recently, Sehgal and Ram [112] mapped the upstream and downstream connectivity within the JNK network to reveal an enrichment of bi-fan and FFL motifs formed immediately upstream and downstream of JNK in addition to negative FBL motifs that exist through transcriptional activation of phosphatases that target the JNK pathway. Shah et al. [113] demonstrated in Sprague-Dawley rats that diet and carcinogen exposure modulated a number of miRNAs (miR-16, miR19b, miR-21, miR26b, miR27b, miR-93, and miR-203) linked to canonical oncogenic signaling pathways. Recently, Song et al. [114] identified a key miRNA regulatory network including miR-200b, miR-200c, and miR-125b that defines the mesenchymal gastric cancer subtype significantly associated with poor overall survival in gastric cancer. Overall, there is very limited information available in the literature on miRNA networks in chemoprevention as the importance of applying network analysis to miRNA chemoprevention research is in its infancy and only beginning to be recognized. Based on our chemoprevention network in Fig. 1d, several miRNA nodes with high "degree" were visible including miR-148b-3p, miR377-3p, miR-103-3p, miR-292-5p, miR-1207-5p, miR-130a3p, miR-92a-3p, miR-16-5p, miR-186-5p, miR-125b-5p, miR-135a-5p, miR-181a-5p, miR-296-5p, miR-200b-3p, miR-21, miR-146a-5p, miR-150-5p, miR-17-5p, miR-2213p, miR-let-7, miR-17, miR-26, miR-192, miR-29, miR193, among others. Further, autoregulation was observed for miR-21, miR-155, and miR-29; also, let-7, miR-17, and miR26 exhibited highest "in-degree," whereas most other miRNA nodes exhibited higher "out-degree" in Fig. 1b. Moreover, miR-31, miR-192, miR-29, and miR-193 showed only "indegree" interactions, whereas miR-17-5p, miR-292-5p, and miR-200b-3p showed only "out-degree" interactions. In addition, several targets of the miRNAs with high "degree" were observed in the network $1 \mathrm{~d}$ including LCOR, BAZ2A, GPATCH8, FRYL, MTF1, DCP1A, GPC6, ZBTB34, FAM19A1, FAM126B, FAM46C, DICER1, EPHB6, 
DDX20, AGO2, E2F3, SLC16A6, CCNJ, TMCC1, HMBOX1, GOLGA1, SBNO1, LIN28A, among others, which may be important in conferring or aiding chemoprotection via miRNA-mediated mechanisms apart from other role(s) in the cellular milieu.

\section{microRNAs and Bifunctional Cancer Networks}

To overcome the limitations of a static protein-protein interaction network, Luo and Kuang [115] have recently proposed a new method to predict essential proteins by integrating dynamic local average connectivity and in-degree of proteins in complexes. Extending network dynamics to miRNA networks, Xue et al. [116] showed recently that negative feedback provided by miR-21 stimulates the propensity of oscillations in NFKB and IL-6 activity, while negative feedback provided by miR-146 dampens the oscillations of NFKB and IL-6, suggesting that variations in the relative strength of the two feedbacks may provide for altered response dynamics to the same stimulus, thus revealing a novel regulatory module of two miRNA-mediated negative feedback loops that allow for the fine tuning of the dynamics of key mediators in inflammation. Schulz et al. [117] developed the MIRna Dynamic Regulatory Events Miner (mirDREM), a probabilistic modeling method that uses input-output hidden Markov models to reconstruct dynamic regulatory networks that explain how temporal gene expression is jointly regulated by miRNAs and transcription factors using postnatal lung development in mice for measurements. Guo et al. [118] noted that miRNA variants (termed isomiRs) are potential functional molecules that may affect miRNA stability or target selection, and multiple isomiR products and miRNA maturation processes provide opportunities to perform versatile roles in the regulatory network, which further enriches and complicates the regulation of biological processes. Collectively, it follows that the cancer cellular milieu is a dynamic environment, and it is probable that same miRNAs may perform different, perhaps opposing, functions at different time points or in different stress conditions, which may explain, at least in part, the bifunctional role of the miRNAs, i.e., both chemoresistant and chemopreventive roles, as depicted in Fig. 1e.

\section{Chemoresistant and Chemopreventive Bifunctional miRNAs}

Using a simple first-principles approach, we analyzed our networks in Fig. 1a and $\mathrm{c}$ to fish out 21 common miRNAs that were present in both chemoresistance and chemopreventive networks, respectively. We then constructed our bifunctional miRNA cancer network Fig. 1e that comprised this common subset of miRNAs that may potentially exert dual functions in both chemoresistance and chemoprevention probably depending on the cellular milieu, temporal status, or stress conditions. These 21 bifunctional miRNAs included let-7, let-7c, let-7e, miR-100, miR-125b, miR-130a, miR-134, miR-15a, miR-16, miR-181a, miR-195, miR-200b, miR-21, miR-215, miR-221, miR-222, miR-224, miR-23a, miR-26a, miR-27b, and miR-342. Figure 1e depicts the architecture of our proposed canonical bifunctional network showing putative interactions between 21 miRNA nodes or vertices involved in both chemoresistance and chemoprevention and 419 undirected edges facilitating these interactions. Further, we queried these miRNAs for interactions with targets to construct an epistemologic bifunctional network showing "arcs" connecting miRNA nodes of the network (Fig. 1f).

\section{Architecture of Bifunctional miRNA Cancer Networks}

Based on our bifunctional network in Fig. 1f, few miRNA nodes with high "degree" were visible, including miR-224-5p, miR-100-5p, miR-200b-3p, miR-26a-5p, let-7a-5p, let-7, miR-27a-3p, miR-23a-3p, miR-125b-5p, miR-16-5p, miR221-3p, miR-342-3p, among others. Further, autoregulation was observed for miR-21, which was found to be modulated by a large number of chemotherapeutic drugs and chemopreventive agents; also, let-7 exhibited highest "in-degree" among nodes, whereas miR-23a-3p, miR-27a-3p, and miR26a-5p exhibited highest "out-degree" among nodes in the network in Fig. 1f. In addition, several targets of the miRNAs with high "degree" were observed in the network in Fig. 1f including SLC4A4, PRTG, TMEM170B, FGF14, EPHB6, CEP85L, UBN2, ZFHX4, WNK3, LIN28B, KBTBD8, CCNJ, TMEM87B, TMEM135, B3GNT1, ARID3A, RBM12B, among others, which may be important in both chemoresistance and chemoprotection functions under appropriate spatial and temporal conditions.

\section{Conclusions}

With new high-throughput technologies and superior computational power available for application to current pharmacology research, biomarker discovery has probably entered its most exciting phase to date, especially with the concurrent advent of systems network biology for "big data." Study of recurrent network motifs in network architecture can inform us better about regulatory pathways in the cellular milieu, more so in complex disease states like cancer with associated co-morbidities and mortality rates. Recently, Di Carlo et al. [119] described a high-level inter-pathway regulatory motif in complex networks called "pathway protection loop" in which miRNAs play a crucial role in the successful behavior and activation of a pathway resulting in new approaches in the identification of therapeutic targets because it could unveil 
novel paths to "activate" or "silence" a target pathway. Indeed, network biology will change the way we look at cellular systems in cancer, and the intrinsic role(s) played by miRNAs will need to be evaluated with a deeper understanding of network dynamics that may confer multiple, sometimes contradictory, roles on signature miRNAs due to spatial and temporal constraints and/or stress conditions. Nazarov et al. [120] investigated simultaneously the transcriptional changes of miRNA and mRNA expression levels using dynamic time series microarray data after activation of the Janus kinase/ signal transducer and activator of transcription (Jak/STAT) pathway by interferon- $\gamma$ stimulation of melanoma cells, and revealed network motifs in the form of FFLs involving transcriptional regulators, mRNAs and miRNAs. Even in other disease states such as HIV, a phased pattern of miRNA expression was evident by next-generation sequencing, and many miRNAs that were initially suppressed were later overexpressed at the height of infection, providing unique signatures of HIV infection [121]. Hwang et al. [122] analyzed regulatory network dynamics in mouse retina to reveal a natural turning point at which the regulatory network of miRNAs, transcription factors, and protein-coding genes undergoes drastic topological changes, thus demonstrating that adding a dynamic dimension to network analysis can provide new insights into retinal development and suggesting that the same approach would likely be useful for the analysis of other developing tissues. Collectively, it is clear that to avoid the limitations of knowledge from static networks, it is important to invest our time and energies in dynamic networks in cancer chemoresistance and chemoprevention in order to make the best informed choice for preventive/therapeutic intervention in cancer. Further, although epigenetic modifications, such as DNA methylation or histone acetylation, have been demonstrated to affect miRNA expression, and to be potentially responsible for the aberrant miRNA regulation observed in cancer, the other side of the coin is represented by the capacity of epi-microRNAs to control the epigenetic machinery directly targeting its enzymatic components [123]. Interestingly, Wang et al. [124] reported that a long noncoding RNA (lncRNA), named mitochondrial dynamic-related lncRNA (MDRL), affects the processing of miR-484 primary transcript in nucleus and regulates the mitochondrial network by targeting miR-361 and miR-484. On the same note, it is certain that miRNA cancer networks are very complex and the regulatory architecture of cancer chemoresistance and/or chemoprevention networks may likely include added dimensions of modulation by epi-miRNAs and lncRNAs, which may further explain, at least in part, the bifunctionality associated with miRNA nodes in our networks apart from temporal dynamics, spatial localization, and stress conditions. Taken together, by a perusal of our chemoresistance, chemoprevention, and bifunctional networks, we can gain deeper insights into the architecture of miRNA regulatory networks in cancer that will serve as the basis for future dynamic network studies and facilitate the discovery of novel miRNA/target biomarkers for preventive and/or therapeutic intervention in cancer.

Acknowledgments This work is supported in part by institutional funds from Amrita University, India and Rutgers University, USA; in part by R01-CA118947, R01-CA152826, from the National Cancer Institute (NCI), R01AT007065 from the National Center for Complementary and Alternative Medicines (NCCAM) and the Office of Dietary Supplements (ODS) to Dr. Ah-Ng Tony Kong; and in part by BT/ PR13157/GBD/27/210/2009 to Dr. Sujit Nair from the Department of Biotechnology, Government of India.

\section{Compliance with Ethics Guidelines}

Conflict of Interest Sujit Nair and Ah-Ng Tony Kong declare that they have no conflict of interest.

Human and Animal Rights and Informed Consent This article does not contain any studies with human or animal subjects performed by any of the authors.

\section{References}

Papers of particular interest, published recently, have been highlighted as:

- Of importance

•. Of major importance

1.• Garofalo M, Croce CM. MicroRNAs as therapeutic targets in chemoresistance. Drug resistance updates: reviews and commentaries in antimicrobial and anticancer chemotherapy 2013, 16(35):47-59. This manuscript discusses the importance of miRNAs in chemoresistance as well as miRNA-based cancer therapeutics.

2. Bottai G, Pasculli B, Calin GA, Santarpia L. Targeting the microRNA-regulating DNA damage/repair pathways in cancer. Expert Opinion on Biological Therapy 2014:1-17.

3. Alshalalfa M. miRNA regulation in the context of functional protein networks: principles and applications. Wiley Interdiscip Rev Syst Biol Med. 2014;6(2):189-99. This manuscript defines principles of miRNA regulation of protein and gene regulatory networks.

4. Yousef M, Trinh HV, Allmer J. Intersection of MicroRNA and gene regulatory networks and their implication in cancer. Curr Pharm Biotechnol. 2014;15(5):445-54.

5. Janky R, Verfaillie A, Imrichova H, Van de Sande B, Standaert L, Christiaens $\mathrm{V}$, et al. iRegulon: from a gene list to a gene regulatory network using large motif and track collections. PLoS Comput Biol. 2014;10(7):e1003731.

6. Mactier KE, Glaire MA, Basavaraju U, El-Omar EM, Hold GL. MicroRNAs in gastrointestinal malignancy: a tool in cancer prevention? European journal of cancer prevention: the official journal of the European Cancer Prevention Organisation 2014;23(6): 540-9.

7. Yi B, Piazza GA, Su X, Xi Y. MicroRNA and cancer chemoprevention. Cancer Prev Res (Phila). 2013;6(5):401-9.

8. Quackenbush J. Extracting biology from high-dimensional biological data. J Exp Biol. 2007;210(9):1507-17.

9. Antman E, Weiss S, Loscalzo J. Systems pharmacology, pharmacogenetics, and clinical trial design in network medicine. Wiley Interdiscip Rev Syst Biol Med. 2012;4(4):367-83. 
10. Bruggeman FJ, Westerhoff HV. The nature of systems biology. Trends Microbiol. 2007;15(1):45-50.

11. Ma'ayan A, Rouillard AD, Clark NR, Wang Z, Duan Q, Kou Y. Lean big data integration in systems biology and systems pharmacology. Trends Pharmacol Sci. 2014;35(9):450-60.

12.•• Nair S, Liew C, Khor TO, Cai L, Kong AN. Differential signaling regulatory networks governing hormone refractory prostate cancers. J Chin Pharm Sci. 2014;23(8):511-24. This manuscript shows different network topologies and network motifs associated with them in androgen-independent prostate cancers.

13.• Nair S, Doh ST, Chan JY, Kong AN, Cai L. Regulatory potential for concerted modulation of Nrf2- and Nfkb1-mediated gene expression in inflammation and carcinogenesis. Br J Cancer. 2008;99(12):2070-82. This manuscript demonstrates a canonical network between Nrf2 and Nfkb1 with putative interactions between the nodes of the network.

14. Mei Q, Li X, Guo M, Fu X, Han W. The miRNA network: microregulator of cell signaling in cancer. Expert Rev Anticancer Ther. 2014;14:1-13.

15. Sun Y, Wang L, Guo SC, Wu XB, Xu XH. High-throughput sequencing to identify miRNA biomarkers in colorectal cancer patients. Oncol Lett. 2014;8(2):711-3.

16. Zhang W, Zang J, Jing X, Sun Z, Yan W, Yang D, et al. Identification of candidate miRNA biomarkers from miRNA regulatory network with application to prostate cancer. J Transl Med. 2014;12:66.

17. Zhu L, Liu J, Liang F, Rayner S, Xiong J. Predicting response to preoperative chemotherapy agents by identifying drug action on modeled microRNA regulation networks. PLoS One. 2014;9(5): e98140.

18. To KK. MicroRNA: a prognostic biomarker and a possible druggable target for circumventing multidrug resistance in cancer chemotherapy. J Biomed Sci. 2013;20:99.

19. Gurtan AM, Sharp PA. The role of miRNAs in regulating gene expression networks. J Mol Biol. 2013;425(19):3582-600.

20. Borotkanics R, Lehmann H. Network motifs that recur across species, including gene regulatory and protein-protein interaction networks. Archives of toxicology 2014.

21. Zhang HM, Kuang S, Xiong X, Gao T, Liu C, Guo AY. Transcription factor and microRNA co-regulatory loops: important regulatory motifs in biological processes and diseases. Briefings in bioinformatics 2013.

22. Liu X, Chen X, Yu X, Tao Y, Bode AM, Dong Z, et al. Regulation of microRNAs by epigenetics and their interplay involved in cancer. J Exp Clin Cancer Res: CR. 2013;32:96.

23. Panczyk M. Pharmacogenetics research on chemotherapy resistance in colorectal cancer over the last 20 years. World J Gastroenterol: WJG. 2014;20(29):9775-827.

24. Suh DH, Kim HS, Kim B, Song YS. Metabolic orchestration between cancer cells and tumor microenvironment as a coevolutionary source of chemoresistance in ovarian cancer: a therapeutic implication. Biochemical pharmacology 2014.

25. Hong L, Han Y, Yang J, Zhang H, Zhao Q, Wu K, et al. MicroRNAs in gastrointestinal cancer: prognostic significance and potential role in chemoresistance. Expert Opin Biol Ther. 2014;14(8):1103-11.

26. Gong Z, Yang J, Li J, Yang L, Le Y, Wang S, et al. Novel insights into the role of microRNA in lung cancer resistance to treatment and targeted therapy. Curr Cancer Drug Targets. 2014;14(3):241-58.

27.• Neelakandan K, Babu P, Nair S. Emerging roles for modulation of microRNA signatures in cancer chemoprevention. Curr Cancer Drug Targets. 2012;12(6):716-40. This manuscript presents several chemotherapeutic drugs and chemopreventive agents as well as miRNAs modulated by them in chemoresistance and chemoprevention respectively in a concise tabular format.

28. Valeri N, Gasparini P, Braconi C, Paone A, Lovat F, Fabbri M, et al. MicroRNA-21 induces resistance to 5-fluorouracil by down- regulating human DNA MutS homolog 2 (hMSH2). Proc Natl Acad Sci U S A. 2010;107(49):21098-103.

29. Borralho PM, Kren BT, Castro RE, da Silva IB, Steer CJ, Rodrigues CM. MicroRNA-143 reduces viability and increases sensitivity to 5-fluorouracil in HCT116 human colorectal cancer cells. FEBS J. 2009;276(22):6689-700.

30. Akao Y, Noguchi S, Iio A, Kojima K, Takagi T, Naoe T. Dysregulation of microRNA-34a expression causes drugresistance to 5-FU in human colon cancer DLD-1 cells. Cancer Lett. 2011;300(2):197-204.

31. Nishida N, Yamashita S, Mimori K, Sudo T, Tanaka F, Shibata K, et al. MicroRNA-10b is a prognostic indicator in colorectal cancer and confers resistance to the chemotherapeutic agent 5fluorouracil in colorectal cancer cells. Ann Surg Oncol. 2012;19(9):3065-71.

32. Yang X, Yin J, Yu J, Xiang Q, Liu Y, Tang S, et al. miRNA-195 sensitizes human hepatocellular carcinoma cells to 5-FU by targeting BCL-w. Oncol Rep. 2012;27(1):250-7.

33. Boni V, Bitarte N, Cristobal I, Zarate R, Rodriguez J, Maiello E, et al. miR-192/miR-215 influence 5-fluorouracil resistance through cell cycle-mediated mechanisms complementary to its post-transcriptional thymidilate synthase regulation. Mol Cancer Ther. 2010;9(8):2265-75.

34. Hao M, Zhang L, An G, Meng H, Han Y, Xie Z, et al. Bone marrow stromal cells protect myeloma cells from bortezomib induced apoptosis by suppressing microRNA-15a expression. Leuk Lymphoma. 2011;52(9):1787-94.

35. Fujita Y, Kojima K, Hamada N, Ohhashi R, Akao Y, Nozawa Y, et al. Effects of miR-34a on cell growth and chemoresistance in prostate cancer PC3 cells. Biochem Biophys Res Commun. 2008;377(1):114-9.

36. Ragusa M, Majorana A, Statello L, Maugeri M, Salito L, Barbagallo D, et al. Specific alterations of microRNA transcriptome and global network structure in colorectal carcinoma after cetuximab treatment. Mol Cancer Ther. 2010;9(12): 3396-409.

37. Hatakeyama H, Cheng H, Wirth P, Counsell A, Marcrom SR, Wood CB, et al. Regulation of heparin-binding EGF-like growth factor by miR-212 and acquired cetuximab-resistance in head and neck squamous cell carcinoma. PLoS One. 2010;5(9):e12702.

38. Sorrentino A, Liu CG, Addario A, Peschle C, Scambia G, Ferlini C. Role of microRNAs in drug-resistant ovarian cancer cells. Gynecol Oncol. 2008;111(3):478-86.

39. Bhatnagar N, Li X, Padi SK, Zhang Q, Tang MS, Guo B. Downregulation of miR-205 and miR-31 confers resistance to chemotherapy-induced apoptosis in prostate cancer cells. Cell Death Dis. 2010;1:e105.

40. Rothschild SI, Tschan MP, Federzoni EA, Jaggi R, Fey MF, Gugger M, et al. MicroRNA-29b is involved in the Src-ID1 signaling pathway and is dysregulated in human lung adenocarcinoma. Oncogene. 2012;31(38):4221-32.

41. Schotte D, De Menezes RX, Akbari Moqadam F, Khankahdani LM, Lange-Turenhout E, Chen C, et al. MicroRNA characterize genetic diversity and drug resistance in pediatric acute lymphoblastic leukemia. Haematologica. 2011;96(5):703-11.

42. Feng B, Wang R, Song HZ, Chen LB. MicroRNA-200b reverses chemoresistance of docetaxel-resistant human lung adenocarcinoma cells by targeting E2F3. Cancer. 2012;118(13):3365-76.

43. Feng B, Wang R, Chen LB. MiR-100 resensitizes docetaxelresistant human lung adenocarcinoma cells (SPC-A1) to docetaxel by targeting Plk1. Cancer Lett. 2012;317(2):184-91.

44. Kovalchuk O, Filkowski J, Meservy J, Ilnytskyy Y, Tryndyak VP, Chekhun VF, et al. Involvement of microRNA-451 in resistance of the MCF-7 breast cancer cells to chemotherapeutic drug doxorubicin. Mol Cancer Ther. 2008;7(7):2152-9. 
45. Henry JC, Park JK, Jiang J, Kim JH, Nagorney DM, Roberts LR, et al. miR-199a-3p targets CD44 and reduces proliferation of CD44 positive hepatocellular carcinoma cell lines. Biochem Biophys Res Commun. 2010;403(1):120-5.

46. Chen J, Tian W, Cai H, He H, Deng Y. Down-regulation of microRNA-200c is associated with drug resistance in human breast cancer. Med Oncol. 2012;29(4):2527-34.

47. Wang ZX, Lu BB, Wang H, Cheng ZX, Yin YM. MicroRNA-21 modulates chemosensitivity of breast cancer cells to doxorubicin by targeting PTEN. Arch Med Res. 2011;42(4):281-90.

48. Bryant JL, Britson J, Balko JM, Willian M, Timmons R, Frolov A, et al. A microRNA gene expression signature predicts response to erlotinib in epithelial cancer cell lines and targets EMT. Br J Cancer. 2012;106(1):148-56.

49. Nagaraja AK, Creighton CJ, Yu Z, Zhu H, Gunaratne PH, Reid JG, et al. A link between mir-100 and FRAP1/mTOR in clear cell ovarian cancer. Mol Endocrinol. 2010;24(2):447-63.

50. Ferracin M, Zagatti B, Rizzotto L, Cavazzini F, Veronese A, Ciccone M, et al. MicroRNAs involvement in fludarabine refractory chronic lymphocytic leukemia. Mol Cancer. 2010;9:123.

51. Moussay E, Palissot V, Vallar L, Poirel HA, Wenner T, El Khoury $\mathrm{V}$, et al. Determination of genes and microRNAs involved in the resistance to fludarabine in vivo in chronic lymphocytic leukemia. Mol Cancer. 2010;9:115.

52. Rao X, Di Leva G, Li M, Fang F, Devlin C, Hartman-Frey C, et al. MicroRNA-221/222 confers breast cancer fulvestrant resistance by regulating multiple signaling pathways. Oncogene. 2011;30(9): 1082-97.

53. Garofalo M, Romano G, Di Leva G, Nuovo G, Jeon YJ, Ngankeu A, et al. EGFR and MET receptor tyrosine kinase-altered microRNA expression induces tumorigenesis and gefitinib resistance in lung cancers. Nat Med. 2012;18(1):74-82.

54. Zhong M, Ma X, Sun C, Chen L. MicroRNAs reduce tumor growth and contribute to enhance cytotoxicity induced by gefitinib in non-small cell lung cancer. Chem Biol Interact. 2010;184(3): 431-8.

55. Iorio MV, Casalini P, Piovan C, Di Leva G, Merlo A, Triulzi T, et al. microRNA-205 regulates HER3 in human breast cancer. Cancer Res. 2009;69(6):2195-200.

56. Dong J, Zhao YP, Zhou L, Zhang TP, Chen G. Bcl-2 upregulation induced by miR-21 via a direct interaction is associated with apoptosis and chemoresistance in MIA PaCa- 2 pancreatic cancer cells. Arch Med Res. 2011;42(1):8-14.

57. Giovannetti E, Funel N, Peters GJ, Del Chiaro M, Erozenci LA, Vasile E, et al. MicroRNA-21 in pancreatic cancer: correlation with clinical outcome and pharmacologic aspects underlying its role in the modulation of gemcitabine activity. Cancer Res. 2010;70(11):4528-38.

58. Park JK, Lee EJ, Esau C, Schmittgen TD. Antisense inhibition of microRNA-21 or -221 arrests cell cycle, induces apoptosis, and sensitizes the effects of gemcitabine in pancreatic adenocarcinoma. Pancreas. 2009;38(7):e190-9.

59. San Jose-Eneriz E, Roman-Gomez J, Jimenez-Velasco A, Garate L, Martin V, Cordeu L, et al. MicroRNA expression profiling in Imatinib-resistant chronic myeloid leukemia patients without clinically significant ABL1-mutations. Mol Cancer. 2009;8:69.

60. Song B, Wang Y, Titmus MA, Botchkina G, Formentini A, Kornmann M, et al. Molecular mechanism of chemoresistance by miR-215 in osteosarcoma and colon cancer cells. Mol Cancer. 2010;9:96.

61. Mencia N, Selga E, Noe V, Ciudad CJ. Underexpression of miR224 in methotrexate resistant human colon cancer cells. Biochem Pharmacol. 2011;82(11):1572-82.

62. Fujita Y, Kojima K, Ohhashi R, Hamada N, Nozawa Y, Kitamoto A, et al. MiR-148a attenuates paclitaxel resistance of hormone- refractory, drug-resistant prostate cancer PC3 cells by regulating MSK1 expression. J Biol Chem. 2010;285(25):19076-84.

63. Shimizu S, Takehara T, Hikita H, Kodama T, Miyagi T, Hosui A, et al. The let-7 family of microRNAs inhibits Bcl-xL expression and potentiates sorafenib-induced apoptosis in human hepatocellular carcinoma. J Hepatol. 2010;52(5):698-704.

64. Berkers J, Govaere O, Wolter P, Beuselinck B, Schoffski P, van Kempen LC, et al. A possible role for microRNA-141 downregulation in sunitinib resistant metastatic clear cell renal cell carcinoma through induction of epithelial-to-mesenchymal transition and hypoxia resistance. J Urol. 2013;189(5):1930-8.

65. Miller TE, Ghoshal K, Ramaswamy B, Roy S, Datta J, Shapiro CL, et al. MicroRNA-221/222 confers tamoxifen resistance in breast cancer by targeting p27Kip1. J Biol Chem. 2008;283(44): 29897-903.

66. Shi L, Chen J, Yang J, Pan T, Zhang S, Wang Z. MiR-21 protected human glioblastoma U87MG cells from chemotherapeutic drug temozolomide induced apoptosis by decreasing Bax/Bcl-2 ratio and caspase-3 activity. Brain Res. 2010;1352:255-64.

67. Ujifuku K, Mitsutake N, Takakura S, Matsuse M, Saenko V, Suzuki K, et al. miR-195, miR-455-3p and miR-10a $\left(^{*}\right)$ are implicated in acquired temozolomide resistance in glioblastoma multiforme cells. Cancer Lett. 2010;296(2):241-8.

68. Shi L, Zhang S, Feng K, Wu F, Wan Y, Wang Z, et al. MicroRNA$125 \mathrm{~b}-2$ confers human glioblastoma stem cells resistance to temozolomide through the mitochondrial pathway of apoptosis. Int $\mathbf{J}$ Oncol. 2012;40(1):119-29.

69. Gong C, Yao Y, Wang Y, Liu B, Wu W, Chen J, et al. Upregulation of miR-21 mediates resistance to trastuzumab therapy for breast cancer. J Biol Chem. 2011;286(21):19127-37.

70. Vera J, Lai X, Schmitz U, Wolkenhauer O. MicroRNA-regulated networks: the perfect storm for classical molecular biology, the ideal scenario for systems biology. Adv Exp Med Biol. 2013;774: 55-76.

71. He D, Gu X, Jiang L, Jin J, Ma X. A methylation-based regulatory network for microRNA 320a in chemoresistant breast cancer. Molecular pharmacology 2014;86(5):536-47.

72. Knoll S, Emmrich S, Putzer BM. The E2F1-miRNA cancer progression network. Adv Exp Med Biol. 2013;774:135-47.

73. Vera J, Schmitz U, Lai X, Engelmann D, Khan FM, Wolkenhauer $\mathrm{O}$, et al. Kinetic modeling-based detection of genetic signatures that provide chemoresistance via the E2F1-p73/DNp73-miR-205 network. Cancer Res. 2013;73(12):3511-24.

74. Kopp F, Oak PS, Wagner E, Roidl A. miR-200c sensitizes breast cancer cells to doxorubicin treatment by decreasing TrkB and Bmil expression. PLoS One. 2012;7(11):e50469.

75. Ratovitski EA. Phospho-DeltaNp63alpha/microRNA network modulates epigenetic regulatory enzymes in squamous cell carcinomas. Cell Cycle. 2014;13(5):749-61.

76. Lecca P. Methods of biological network inference for reverse engineering cancer chemoresistance mechanisms. Drug Discov Today. 2014;19(2):151-63.

77. Seo H, Kim W, Lee J, Youn B. Network-based approaches for anticancer therapy (Review). Int J Oncol. 2013;43(6):1737-44.

78. Sporn MB, Suh N. Chemoprevention of cancer. Carcinogenesis. 2000;21(3):525-30.

79. Nair S, Li W, Kong AN. Natural dietary anti-cancer chemopreventive compounds: redox-mediated differential signaling mechanisms in cytoprotection of normal cells versus cytotoxicity in tumor cells. Acta Pharmacol Sin. 2007;28(4):459-72. This manuscript reviews several natural dietary factors that are important in chemoprevention.

80.• Li Y, Kong D, Wang Z, Sarkar FH. Regulation of microRNAs by natural agents: an emerging field in chemoprevention and chemotherapy research. Pharm Res. 2010;27(6):1027-41. This manuscript suggests that specific targeting of miRNAs by natural agents 
could kill drug-resistant cells and improve survival outcomes in patients with cancer.

81. Kong AN, Zhang C, Su ZY. Targeting epigenetics for cancer prevention by dietary cancer preventive compounds - the case of miRNA. Cancer Prev Res (Phila). 2013;6(7):622-4.

82. Garzon R, Pichiorri F, Palumbo T, Visentini M, Aqeilan R, Cimmino A, et al. MicroRNA gene expression during retinoic acid-induced differentiation of human acute promyelocytic leukemia. Oncogene. 2007;26(28):4148-57.

83. Rossi A, D'Urso OF, Gatto G, Poltronieri P, Ferracin M, Remondelli P, et al. Non-coding RNAs change their expression profile after retinoid induced differentiation of the promyelocytic cell line NB4. BMC Res Notes. 2010;3:24.

84. Wagner AE, Boesch-Saadatmandi C, Dose J, Schultheiss G, Rimbach G. Anti-inflammatory potential of allyl-isothiocyanate-role of Nrf2, NF-(kappa) B and microRNA-155. J Cell Mol Med. 2012;16(4):836-43.

85. Takahashi M, Sung B, Shen Y, Hur K, Link A, Boland CR, et al. Boswellic acid exerts antitumor effects in colorectal cancer cells by modulating expression of the let-7 and miR-200 microRNA family. Carcinogenesis. 2012;33(12):2441-9.

86. Wang X, Gocek E, Liu CG, Studzinski GP. MicroRNAs181 regulate the expression of p27Kip1 in human myeloid leukemia cells induced to differentiate by 1,25-dihydroxyvitamin D3. Cell Cycle. 2009;8(5):736-41.

87. Essa S, Denzer N, Mahlknecht U, Klein R, Collnot EM, Tilgen W, et al. VDR microRNA expression and epigenetic silencing of vitamin D signaling in melanoma cells. J Steroid Biochem Mol Biol. 2010;121(1-2):110-3.

88. Wang WL, Chatterjee N, Chittur SV, Welsh J, Tenniswood MP. Effects of 1alpha,25 dihydroxyvitamin D3 and testosterone on miRNA and mRNA expression in LNCaP cells. Mol Cancer. 2011;10:58

89. Sun M, Estrov Z, Ji Y, Coombes KR, Harris DH, Kurzrock R. Curcumin (diferuloylmethane) alters the expression profiles of microRNAs in human pancreatic cancer cells. Mol Cancer Ther. 2008;7(3):464-73

90. Zhang J, Du Y, Wu C, Ren X, Ti X, Shi J, et al. Curcumin promotes apoptosis in human lung adenocarcinoma cells through miR-186* signaling pathway. Oncol Rep. 2010;24(5):1217-23.

91. Zhang J, Zhang T, Ti X, Shi J, Wu C, Ren X, et al. Curcumin promotes apoptosis in A549/DDP multidrug-resistant human lung adenocarcinoma cells through an miRNA signaling pathway. Biochem Biophys Res Commun. 2010;399(1):1-6.

92. Mudduluru G, George-William JN, Muppala S, Asangani IA, Kumarswamy R, Nelson LD, et al. Curcumin regulates miR-21 expression and inhibits invasion and metastasis in colorectal cancer. Biosci Rep. 2011;31(3):185-97.

93. Ali S, Ahmad A, Banerjee S, Padhye S, Dominiak K, Schaffert JM, et al. Gemcitabine sensitivity can be induced in pancreatic cancer cells through modulation of miR-200 and miR-21 expression by curcumin or its analogue CDF. Cancer Res. 2010;70(9):3606-17.

94. Yang J, Cao Y, Sun J, Zhang Y. Curcumin reduces the expression of Bcl-2 by upregulating miR-15a and miR-16 in MCF-7 cells. Med Oncol. 2010;27(4):1114-8.

95. Chiang EP, Chiu SC, Pai MH, Wang YC, Wang FY, Kuo YH, et al. Organosulfur garlic compounds induce neovasculogenesis in human endothelial progenitor cells through a modulation of microRNA 221 and the PI3-K/Akt signaling pathways. J Agric Food Chem. 2013;61(20):4839-49.

96. Li Y, VandenBoom 2nd TG, Kong D, Wang Z, Ali S, Philip PA, et al. Up-regulation of miR-200 and let-7 by natural agents leads to the reversal of epithelial-to-mesenchymal transition in gemcitabine-resistant pancreatic cancer cells. Cancer Res. 2009;69(16):6704-12
97. Li Y, 2nd Vandenboom TG, Wang Z, Kong D, Ali S, Philip PA, et al. miR-146a suppresses invasion of pancreatic cancer cells. Cancer Res. 2010;70(4):1486-95.

98. Wen XY, Wu SY, Li ZQ, Liu ZQ, Zhang JJ, Wang GF, et al. Ellagitannin (BJA3121), an anti-proliferative natural polyphenol compound, can regulate the expression of MiRNAs in HepG2 cancer cells. Phytother Res: PTR. 2009;23(6):778-84.

99. Ahn J-I, Jeong K, Ko M-J, Shin H, Kim H, Chung H, et al. Changes of miRNA and mRNA expression in HepG2 cells treated by epigallocatechin gallate. Mol Cell Toxicol. 2010;6(2):169-77.

100. Tsang WP, Kwok TT. Epigallocatechin gallate up-regulation of miR-16 and induction of apoptosis in human cancer cells. J Nutr Biochem. 2010;21(2):140-6.

101. Siddiqui IA, Asim M, Hafeez BB, Adhami VM, Tarapore RS, Mukhtar H. Green tea polyphenol EGCG blunts androgen receptor function in prostate cancer. FASEB J: Off Publ Fed Am Soc Exp Biol. 2011;25(4):1198-207.

102. Sun Q, Cong R, Yan H, Gu H, Zeng Y, Liu N, et al. Genistein inhibits growth of human uveal melanoma cells and affects microRNA-27a and target gene expression. Oncol Rep. 2009;22(3):563-7.

103. Fix LN, Shah M, Efferth T, Farwell MA, Zhang B. MicroRNA expression profile of MCF-7 human breast cancer cells and the effect of green tea polyphenon-60. Cancer Genomics Proteomics. 2010;7(5):261-77.

104. Melkamu T, Zhang X, Tan J, Zeng Y, Kassie F. Alteration of microRNA expression in vinyl carbamate-induced mouse lung tumors and modulation by the chemopreventive agent indole-3carbinol. Carcinogenesis. 2010;31(2):252-8.

105.• Sethi S, Li Y, Sarkar FH. Regulating miRNA by natural agents as a new strategy for cancer treatment. Curr Drug Targets. 2013;14(10):1167-74. This manuscript suggests that natural agents can inhibit cancer progression, increase drug sensitivity, reverse epithelial-to-mesenchymal transition or cause death of cancer stem cells, and prevent metastasis through modulation of miRNAs.

106. Ahn J, Lee H, Jung CH, Ha T. Lycopene inhibits hepatic steatosis via microRNA-21-induced downregulation of fatty acid-binding protein 7 in mice fed a high-fat diet. Mol Nutr Food Res. 2012;56(11):1665-74.

107. Izzotti A, Larghero P, Cartiglia C, Longobardi M, Pfeffer U, Steele VE, et al. Modulation of microRNA expression by budesonide, phenethyl isothiocyanate and cigarette smoke in mouse liver and lung. Carcinogenesis. 2010;31(5):894-901.

108. Boesch-Saadatmandi C, Loboda A, Wagner AE, Stachurska A, Jozkowicz A, Dulak J, et al. Effect of quercetin and its metabolites isorhamnetin and quercetin-3-glucuronide on inflammatory gene expression: role of miR-155. J Nutr Biochem. 2011;22(3):293-9.

109. Dhar S, Hicks C, Levenson AS. Resveratrol and prostate cancer: promising role for microRNAs. Mol Nutr Food Res. 2011;55(8): 1219-29.

110. Tili E, Michaille JJ, Adair B, Alder H, Limagne E, Taccioli C, et al. Resveratrol decreases the levels of miR- 155 by upregulating miR663, a microRNA targeting JunB and JunD. Carcinogenesis. 2010;31(9):1561-6.

111. Appari M, Babu KR, Kaczorowski A, Gross W, Herr I. Sulforaphane, quercetin and catechins complement each other in elimination of advanced pancreatic cancer by miR-let-7 induction and K-ras inhibition. International journal of oncology 2014:45(4):1391-400.

112. Sehgal V, Ram PT. Network motifs in JNK signaling. Genes Cancer. 2013;4(9-10):409-13.

113. Shah MS, Schwartz SL, Zhao C, Davidson LA, Zhou B, Lupton $\mathrm{JR}$, et al. Integrated microRNA and mRNA expression profiling in 
a rat colon carcinogenesis model: effect of a chemo-protective diet. Physiol Genomics. 2011;43(10):640-54.

114. Song F, Yang D, Liu B, Guo Y, Zheng H, Li L, et al. Integrated microRNA network analyses identify a poor-prognosis subtype of gastric cancer characterized by the miR-200 family. Clin Cancer Res: Off J Am Assoc Cancer Res. 2014;20(4):878-89.

115. Luo J, Kuang L. A new method for predicting essential proteins based on dynamic network topology and complex information. Comput Biol Chem. 2014;52C:34-42.

116. Xue X, Xia W, Wenzhong H. A modeled dynamic regulatory network of NF-kappaB and IL-6 mediated by miRNA. Bio Systems. 2013;114(3):214-8.

117. Schulz MH, Pandit KV, Lino Cardenas CL, Ambalavanan N, Kaminski N, Bar-Joseph Z. Reconstructing dynamic microRNAregulated interaction networks. Proc Natl Acad Sci U S A. 2013;110(39):15686-91

118. Guo L, Zhao Y, Yang S, Zhang H, Chen F. A genome-wide screen for non-template nucleotides and isomiR repertoires in miRNAs indicates dynamic and versatile microRNAome. Molecular biology reports 2014;41(10):6649-58.

119. Di Carlo S, Politano G, Savino A, Benso A. A systematic analysis of a mi-RNA inter-pathway regulatory motif. J Clin Bioinforma. 2013;3(1):20.

120. Nazarov PV, Reinsbach SE, Muller A, Nicot N, Philippidou D, Vallar L, et al. Interplay of microRNAs, transcription factors and target genes: linking dynamic expression changes to function. Nucleic Acids Res. 2013;41(5):2817-31.

121. Chang ST, Thomas MJ, Sova P, Green RR, Palermo RE, Katze MG. Next-generation sequencing of small RNAs from HIVinfected cells identifies phased microrna expression patterns and candidate novel microRNAs differentially expressed upon infection. mBio. 2013;4(1):e00549-512.

122. Hwang W, Hackler Jr L, Wu G, Ji H, Zack DJ, Qian J. Dynamics of regulatory networks in the developing mouse retina. PLoS One. 2012;7(10):e46521.

123. Iorio MV, Piovan C, Croce CM. Interplay between microRNAs and the epigenetic machinery: an intricate network. Biochim Biophys Acta. 2010;1799(10-12):694-701.

124. Wang K, Sun T, Li N, Wang Y, Wang JX, Zhou LY, et al. MDRL IncRNA regulates the processing of miR-484 primary transcript by targeting miR-361. PLoS Genet. 2014;10(7):e1004467.

125. Saito R, Smoot ME, Ono K, Ruscheinski J, Wang PL, Lotia S, et al. A travel guide to Cytoscape plugins. Nat Methods. 2012;9(11):1069-76.

126. Cline MS, Smoot M, Cerami E, Kuchinsky A, Landys N, Workman $\mathrm{C}$, et al. Integration of biological networks and gene expression data using Cytoscape. Nat Protoc. 2007;2(10): 2366-82. 